Document downloaded from:

http://hdl.handle.net/10251/61536

This paper must be cited as:

Baselga Moreno, S.; García-Asenjo Villamayor, L.; Garrigues Talens, P. (2014). Practical formulas for the refraction coefficient. Journal of Surveying Engineering. 140(2):1-5. doi:10.1061/(ASCE)SU.1943-5428.0000124.

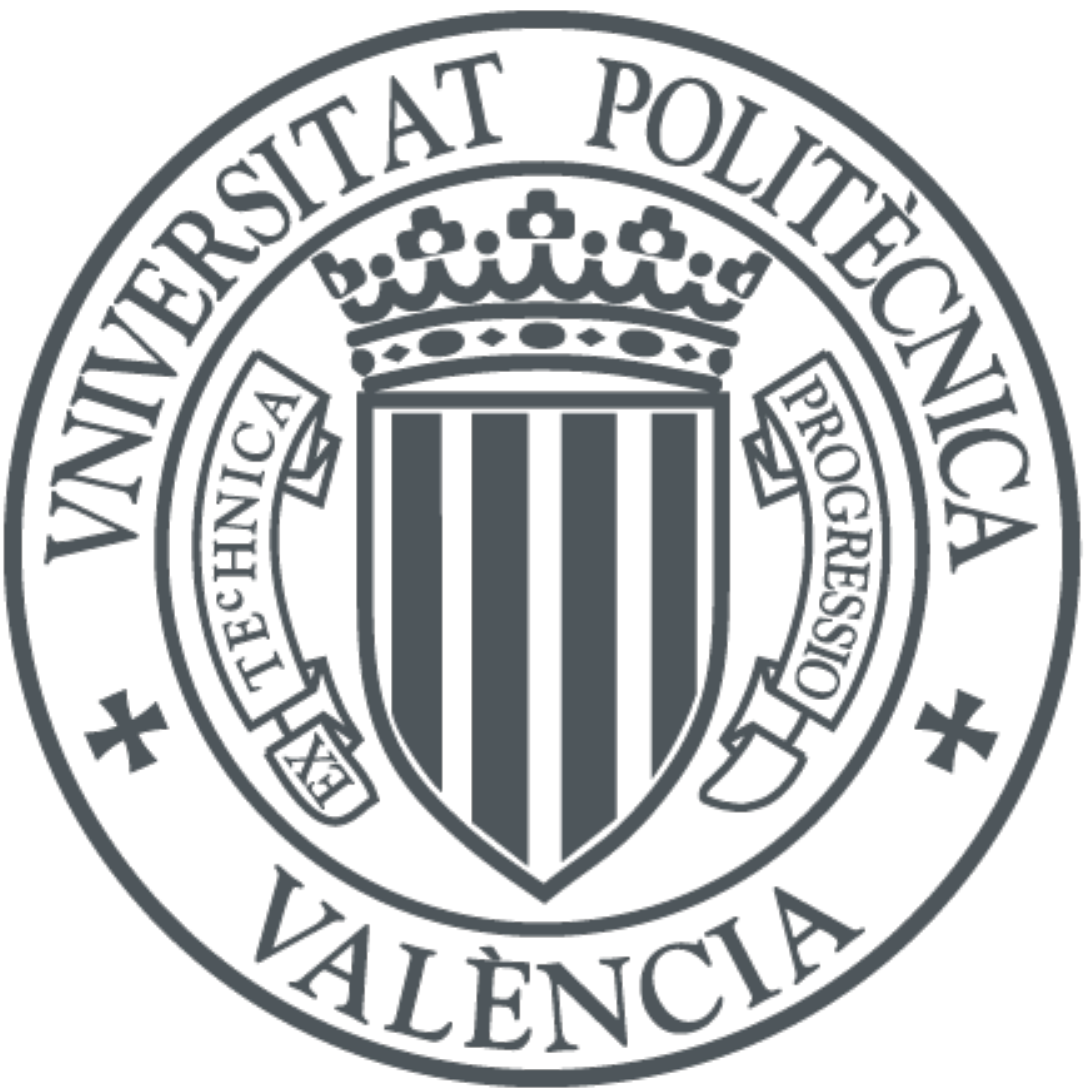

The final publication is available at

http://dx.doi.org/10.1061/(ASCE)SU.1943-5428.0000124

Copyright American Society of Civil Engineers

Additional Information 


\title{
Practical Formulas for the Refraction Coefficient
}

\author{
Sergio Baselga M.ASCE ${ }^{1}$, Luis García-Asenjo ${ }^{2}$ and Pascual Garrigues ${ }^{3}$
}

Abstract: Knowledge of the actual refraction coefficient is essential in leveling surveys and precise electromagnetic distance measurement reduction. The most common method followed by the surveyor for its determination is based on the use of simultaneous reciprocal zenith observations. Given that the commonly used formula is only an approximation valid for approximately horizontal sightings, in a recent work the exact geometric solution was obtained. However, the closed form expression for the solution turned out to be very complicated so that an iterative computation procedure was suggested instead. In the present paper, we want to derive from the complete solution a compact formula that is easy to implement and retains the necessary accuracy for horizontal and slanted sightings. In addition, we will also focus on the common situation for the surveyor where isolated observations have to be done and no partially compensating procedures - e.g. leap-frog or middle point - are possible. If temperature vertical profiles are unknown then the refraction coefficient cannot be reliably determined. Some surveyors may customarily use then an average value, e.g. $k=0.13$, perhaps being unaware of the risks involved in such simplistic assumption. In the present paper, we also want to present a useful and simple formula for approximately estimating the refraction coefficient in terms of easily accessible parameters in order to correct the bulk of the refraction effect in single observations, always bearing in mind that determination of the refraction coefficient by means of a model may turn out to be some inaccurate, but still better than the blind use of a universal $k$.

CE Database subject headings: Refraction; Leveling; Geodetic surveys.

\footnotetext{
${ }^{1}$ (Corresponding author) Lecturer, Dpto. Ingeniería Cartográfica, Geodesia y Fotogrametría, Universidad Politécnica de Valencia, Camino de Vera s/n, 46022 Valencia, Spain. Email: serbamo@cgf.upv.es.

2 Lecturer, Dpto. Ingeniería Cartográfica, Geodesia y Fotogrametría, Universidad Politécnica de Valencia, Camino de Vera s/n, 46022 Valencia, Spain. Email: lugarcia@cgf.upv.es.

3 Lecturer, Dpto. Ingeniería Cartográfica, Geodesia y Fotogrametría, Universidad Politécnica de Valencia, Camino de Vera s/n, 46022

Valencia, Spain. Email: pasgarta@cgf.upv.es.
} 


\section{Introduction}

The study and characterization of the light path through the lower atmosphere commonly involved in the near-ground geodetic measurements, usually referred to as geodetic refraction, has occupied scientists for centuries. In the 19th century adoption of a spherical approximation for the actual ray path was proposed and subsequently applied in common geodetic practice. The refraction coefficient $k$ was then defined as the ratio between a mean radius of the earth $R$ and the light path curvature radius $r$

$$
k=\frac{R}{r}
$$

and an approximate value of $k=0.13$ was found and extensively used by Gauss in his computation of the Hanover geodetic network (Brunner 1984). The use of a standard value for the refraction coefficient, however, has proven to be a very limited approximation since it corresponds only to average adiabatic conditions which are particularly not representative of most engineering type measurements made close to the ground where heating effects predominate (Dodson and Zaher, 1985). The use of the actual refraction coefficient is therefore a crucial need not only for leveling but also for applying the necessary reductions in precise electromagnetic distance measurements (EDM). Apart from some more recent proposals, e.g. turbulence determination and dispersometry (Ingensand 2008), the surveyor has classically followed one of two approaches: determination by means of simultaneous reciprocal observations and determination by means of a model for the lower atmosphere.

Observation of simultaneous reciprocal zenith observations is the easiest and most precise method at hand for the surveyor. However, the formula traditionally used is only an approximation valid for approximately horizontal sightings; therefore a rigorous expression shall be preferred in general. Tsoulis et al. (2008) obtained the exact geometric solution for

51 the refraction coefficient by simultaneous reciprocal zenith observations. As the direct 
computation of the exact solution was found to be rather complicated they also derived a more handy iterative procedure. In the present paper, we also want to contribute to this theory by deriving from their complete geometric solution a compact formula that is easy to implement and successfully retains the necessary accuracy for both horizontal and slanted sightings.

On the other hand, determination of the refraction coefficient by means of a model for the lower atmosphere was found to be possible after the work of Kukkamäki (1938). He found that refraction was mainly due to the temperature vertical gradient and proposed a temperature model of the form

$$
T=a+b z^{c}
$$

where $T$ is the temperature, $z$ is the height above the ground, and $a, b$ and $c$ are parameters to be experimentally deduced, which in practice implied the need for measuring temperatures at some different heights above ground. Webb (1969) was the first to introduce in geodesy the idea that temperature gradients could be successfully computed in terms of other meteorological parameters, mainly the upward heating flux, whereas recognizing the practical difficulty in estimating them with acceptable accuracy. Tens of proposals have been given ever since in terms of disparate parameters (intensity of the sun's radiation, wind velocity, cloud cover, surface type and wetness, etc). At any rate, all of them yield a relatively low accuracy for the usual situation for the surveyor where single observations are taken and temperature vertical differences are not directly measured, so that normally it is simply recommended to avoid isolated leveling measurements (Kharaghani, 1987). Obviously, if simultaneous reciprocal observations are not possible one should try to resort to a procedure that cancels first order refraction effects, such as middle point geometric leveling or trigonometric leveling by the leap-frog method (Ceylan and Baykal, 2006), but since this is not always possible a handy formula estimating refraction effects may be welcome. In this 
spirit, we also want to derive in the present paper a simple formula for estimating the refraction coefficient in terms of easily accessible parameters in order to approximately correct refraction in single leveling observations.

\section{Simultaneous reciprocal observations}

Following Tsoulis et al. (2008), the refraction coefficient can be obtained in terms of the refraction angle $\delta$ by

$$
k=\frac{2 R}{s} \sin \delta
$$

where $R$ is a mean radius of the earth, acting as a good approximation to the curvature radius of the equipotential surface, and $s$ is the distance along the straight line between the visual endpoints A and B (chord). Both astronomic verticals form an angle $\gamma$ that according to Tsoulis et al. (2008) derivation fulfills

$$
\sin \gamma=\frac{s}{R} \sin \left(\frac{1}{2}\left(\pi-\gamma+Z_{A}{ }^{\prime}-Z_{B}{ }^{\prime}\right)\right)
$$

where $Z_{A}{ }^{\prime}$ and $Z_{B}{ }^{\prime}$ are the observed simultaneous reciprocal zenith angles (affected by refraction).

Upon determination of $\gamma$, refraction angle $\delta$ and refraction coefficient $k$ can be obtained by

$$
\begin{aligned}
& \delta=\frac{1}{2}\left(\pi+\gamma-Z_{A}{ }^{\prime}-Z_{B}{ }^{\prime}\right) \\
& k=\frac{2 R}{S} \sin \left(\frac{1}{2}\left(\pi+\gamma-Z_{A}{ }^{\prime}-Z_{B}{ }^{\prime}\right)\right)
\end{aligned}
$$

$$
\text { However, Eq. (4) is a nonlinear equation with respect to the unknown } \gamma \text { appearing in both }
$$
sides of the equation. Tsoulis et al. (2008) showed that the exact solution can be obtained by solving a fourth degree equation whose numerical implementation is not simple to program 
99

100

101

102

103

$$
k=\frac{2 R}{S} \sin \left(\frac{1}{2}\left(\pi+\arcsin \left(\frac{s}{R} \sin \left(\frac{1}{2}\left(\pi-\gamma+Z_{A^{\prime}}-Z_{B}{ }^{\prime}\right)\right)\right)-Z_{A^{\prime}}-Z_{B}^{\prime}\right)\right)
$$

104 Second, we can easily see in Eq. (4) that we can substitute the sine of the angle $\gamma$ for the

105 angle itself in the left side of the equation since it is a small angle, and use $\gamma=\frac{S}{R}$ as a fairly 106 good approximation since the sine appearing in the right side of Eq. (4) tends to unity for 107 small $\gamma$ and similar zenith angles.

108 If we plug this approximation $\gamma=\frac{s}{R}$ into Eq. (7) and denote by $f$ the sine function that 109 multiplies $\frac{2 R}{s}$ the expression can be rewritten as

$$
k=\frac{2 R}{s} f\left(\frac{s}{R}\right)
$$

111 and since $\frac{S}{R}$ is small enough

$$
f\left(\frac{s}{R}\right) \approx f(0)+f^{\prime}(0) \frac{s}{R}
$$

The resulting expression is

$$
k=\cos \left(\frac{Z_{A}^{\prime}-Z_{B}^{\prime}}{2}\right) \sin \left(\frac{Z_{A}{ }^{\prime}+Z_{B}{ }^{\prime}}{2}\right)+\frac{2 R}{s} \cos \left(\frac{Z_{A}{ }^{\prime}+Z_{B}{ }^{\prime}}{2}\right)
$$

115 or alternatively

$$
k=\frac{1}{2} \sin Z_{A}{ }^{\prime}+\frac{1}{2} \sin Z_{B}{ }^{\prime}+\frac{2 R}{s} \cos \left(\frac{Z_{A}{ }^{\prime}+Z_{B}{ }^{\prime}}{2}\right)
$$


117 where we can see that usage of the following equality and approximation $118 \cos \left(\frac{Z_{A}{ }^{\prime}+Z_{B}{ }^{\prime}}{2}\right)=-\sin \left(\frac{Z_{A}{ }^{\prime}+Z_{B}{ }^{\prime}-\pi}{2}\right) \approx-\frac{Z_{A}{ }^{\prime}+Z_{B}{ }^{\prime}-\pi}{2}$ in the last term of the right side, and

$119 Z_{A}{ }^{\prime} \approx Z_{B}{ }^{\prime} \approx \frac{\pi}{2}$ in the first and second term of the right side, leads to the commonly used approximate expression

$$
k=1-\frac{R}{S}\left(Z_{A}{ }^{\prime}+Z_{B}{ }^{\prime}-\pi\right)
$$

Table 1 compares the results obtained after application of the different formulas. As it was done in Tsoulis et al. (2008) only for the purpose of facilitating comparisons with respect to a round k-value, given a selected zenith angle at station A and a distance chord, the zenith angle at station B is assigned a value so as to produce the desired k-value (we will focus here only on two representative extreme k-values: -0.15 and 0.40 ).

As it can be seen, the commonly used Eq. (12) performs unsuccessfully for sufficiently slanted sightings whereas our proposed closed expression Eq. (11) provides results with sufficient accuracy and considerably less effort than the rigorous iterative or exact procedure given in Tsoulis et al (2008), which involves Eq. (4) and Eq. (6).

\section{Single observations}

For those situations in which isolated observations are mandatory, both for leveling works and EDM measurements, some recommendations may be welcome - short observations, typically of a maximum of $50 \mathrm{~m}$, with a minimum clearance above ground of typically $50 \mathrm{~cm}$, etc. but there still remains the need for applying a correction model (Brunner 1984).

139 The curvature of vertical refraction, $1 / r$ in Eq. (1), can be substituted by minus the vertical 140 gradient of the refraction index $n$ yielding 


$$
k=-R \frac{d n}{d z}
$$

142 Adoption of the expression for the refraction index recommended in the IAG resolution, Gen.

143 Ass. Birmingham 1999, leads to

$$
k=10^{-6} R\left(78 \frac{p}{T^{2}}\left(0.034+\frac{d T}{d z}\right)+\frac{11}{T} \frac{d e}{d z}\right)
$$

145 for visible and infrared light with temperature $T$ in $\mathrm{K}$, and atmospheric pressure $p$ and water 146 vapour pressure $e$ in hPa for height $z$ in m (Torge 2001). After neglecting the last term and 147 substituting a suitable mean radius it is common to use the following expression (e.g. Hirt et 148 al 2010)

$$
k=503 \frac{p}{T^{2}}\left(0.034+\frac{d T}{d z}\right)
$$

150 where the actual temperature vertical gradient needs to be measured (preferably) or estimated.

151 Standard conditions, including the average temperature gradient in the troposphere $d T / d z \approx-0.0065 \mathrm{~K} / \mathrm{m}$, may enable us to compute a mean value for $k$. This value, however,

153 often happens to be very inaccurate, inasmuch as existing conditions for a sufficiently close to

154 the ground sighting may differ considerably from the troposphere average conditions.

155 Therefore, for the common situation for the surveyor where measured temperature vertical 156 profiles are not available the use of a suitable temperature vertical gradient model is strongly 157 recommended.

158 After the work of Kukkamäki (1938), Webb and Angus-Leppan advanced research 159 subsequently extended by Brunner - to find a relationship between the temperature gradient 160 and the upward heat flux $H$ (Brunner 1984)

$$
\frac{d T}{d z}=-0.027 H^{2 / 3} z^{-4 / 3}
$$


162 The main difficulty was to estimate this heat flux $H$ to a reasonable accuracy without 163 involving expensive instruments and too delicate measurements.

164 Holdahl (1981) used historical records of solar radiation, precipitation, cloud cover and 165 ground reflectivity from hundreds of stations in the conterminous United States to develop a 166 season and location complete model for the temperature gradient (in the form of Kukkamäki 167 Eq. (2) with exponent $c=-1 / 3$ ) and compute precise refraction corrections. Holdahl's model 168 has ever since been used by the National Geodetic Survey (NGS) to compute refraction 169 corrections for historic levelling data for which temperature differences were not observed. Its 170 use, however, is limited to the area were historic data was collected (conterminous United 171 States).

172 On the other hand, micrometeorologist Tait (1949) found a temperature model for the lower 173 atmosphere in terms of the height ratio logarithm.

$$
T_{1}-T_{2}=(0.4-4 I) \log _{10}\left(z_{1} / z_{2}\right)
$$

where $I$ is the horizontal component of total insolation.

176 Recently, Georgakis et al (2010) applied the model to urban canyons and found a useful relationship for heights above ground in the range of 2 to $15 \mathrm{~m}$ introducing the angle of sun 178 elevation $a$ and a value $N$ representing the absence of cloudiness (from $N=0$ for a completely 179 covered sky to $N=1$ for a completely clear sky)

$$
T_{1}-T_{2}=N(1.2-6.8 \sin a) \log _{10}\left(z_{1} / z_{2}\right)
$$

181 Beyond its ease of use, this formula effectively introduces insolation contribution by 182 consideration of the cloud cover percentage and the sun elevation (obviously dependent on 183 location and year season). Our proposal in this paper is to adapt this formula to the low 184 heights above ground usually encountered in surveying practice. We can denote by $A$ and $B$ 185 the coefficients to be experimentally determined and write

$$
T_{1}-T_{2}=N(A-B \sin a) \log _{10}\left(z_{1} / z_{2}\right)
$$


187 Using the direct temperature determinations obtained by Kharaghani (1987) for different 188 heights over different surfaces we found $A$ values to be not significant (close to zero and 189 considerably smaller than their respective uncertainties) and $B$ values to be significant and 190 quite similar: $1.70(\sigma=0.11)$ over gravel, $1.91(\sigma=0.11)$ over grass, and $1.65(\sigma=0.11)$ 191 over asphalt. Even a single adjustment for all surfaces gave a significant value: $192 B=1.70(\sigma=0.06)$ with an average temperature residual of $0.26^{\circ} \mathrm{C}$.

193 The model results in

$$
T_{1}-T_{2}=-B N \sin a \log _{10}\left(z_{1} / z_{2}\right)
$$

195 Substitution of $z_{1}=z+d z$ (for $T_{1}=T+d T$ ) and $z_{2}=z$ (for $T_{2}=T$ ), and first order 196 expansion of the logarithm around unity yields

$$
\begin{gathered}
d T=-B N \sin a \log _{10}\left(1+\frac{d z}{z}\right) \approx-B N \sin a 0.4343 \frac{d z}{z} \\
\frac{d T}{d z} \approx-0.4343 B N \frac{1}{z} \sin a
\end{gathered}
$$

Eq. (15) can now be rewritten as

$$
k=503 \frac{p}{T^{2}}\left(0.034-0.4343 B N \frac{1}{Z} \sin a\right)
$$

where a value $B=1.70$ was empirically found.

Eq. (23) is meant to be only a simple correction accounting for the main bulk of the refraction effect for the unfavourable cases where neither reciprocal simultaneous observations nor partially compensating observation procedures (leap-frog method in trigonometric levelling or middle point in geometric levelling) are available. The extent of its validity has to be further tested. As an application example we give now the results for a 24-h observation campaign were reciprocal simultaneous zenith angles were measured every half an hour with a pair of cover ratio) for a line of $4385.06 \mathrm{~m}$ with an average clearance above ground of $7 \mathrm{~m}$. Fig. 1 
shows the refraction coefficient variation obtained by reciprocal simultaneous angles Eq. (11)

211 in comparison with the values derived by the model Eq. (23) with $B=1.70$. As it can be seen

212 the model serves the desired purpose of coarsely capturing the main refraction trend. On the

213 other hand, any average value like $k=0.13$ results, as expected, completely insufficient.

\section{Figure 1}

215

216

217

218

219

220

221

222

223

224

\section{Conclusions}

A compact formula for computation of the refraction coefficient after reciprocal simultaneous observations was given and shown to be coincident in practice with the exact solution that has to be computed by a complicated algebraic method or an iterative procedure. In addition, for the frequent cases of single observations where refraction effects cannot be partially compensated or accurately computed (since e.g. temperature vertical gradients are not measured) a simple formula has been derived and shown to successfully approximate the bulk of the refraction effect. Further research has to be conducted, however, in order to thoroughly determine the extent of its validity as well as the suitability of the experimentally obtained value for the temperature gradient coefficient.

\section{Acknowledgements}

The authors are grateful to the editor and the anonymous reviewers for their valuable suggestions, corrections and comments that helped improve the original manuscript. This research is funded by the Spanish Ministry of Science and Innovation (AYA2011-23232).

\section{References}

Brunner, F. K. (1984). Geodetic refraction. Effects of electromagnetic wave propagation through the atmosphere, Springer-Verlag, Berlin, Heidelberg. 
237 Ceylan, A., and Baykal, O. (2006). "Precise height determination using leap-frog 238 trigonometric levelling.” J. Surv. Eng., 132(3), 118-123.

239 Dodson, A. H., and Zaher, M. (1985). "Refraction effects on vertical angle measurements.” 240 Surv. Rev., 28(217), 169-183.

241 Georgakis, C., Santamouris, M., and Kaisarlis, G. (2010). "The vertical stratification of air 242 temperature in the center of Athens.” J. Appl. Meteorol. Climatol., 49(6), 1219-1232.

243 Hirt C., Guillaume, S., Wisbar, A., Bürki, B., and Sternberg, H. (2010). "Monitoring of the 244 refraction coefficient in the lower atmosphere using a controlled set-up of simultaneous 245 reciprocal vertical angle measurements.” J. Geophys. Res., 115, D21102.

246 Holdahl, S. R. (1981). “A model of temperature stratification for correction of leveling 247 refraction.” NOAA Tech. Memo. NOS NGS 31, Rockville, Md.

248 Ingensand, H. (2008). "Concepts and solutions to overcome the refraction problem in 249 terrestrial precision measurements.” Geodesy and Cartography, 34(2), 61-65.

250 Kharaghani, G. A. (1987). "Propagation of refraction errors in trigonometric height traversing 251 and geodetic levelling.” Tech. Rep. No. 132, Dept. of Surveying Engineering, University of 252 New Brunswick, Fredericton, N.B.

253 Kukkamäki, T. J. (1938). “Uber die nivellitische refraction.” Finnish Geodetic Institute Pub. 254 No. 25, Helsinki.

255 Tait, G. W. C. (1949). “The vertical temperature gradient in the lower atmosphere under 256 daylight conditions.” Quart. J. Roy. Meteor. Soc., 75, 287-292.

257 Torge, W. (2001). Geodesy, $3^{\text {rd }}$ Ed., de Gruyter, Berlin.

258 Tsoulis, D., Petrovic, S., and Kilian, N. (2008). "Theoretical and numerical aspects of the 259 geodetic method for determining the atmospheric refraction coefficient using simultaneous 260 and mutual zenith observations.” J. Surv. Eng., 134(1), 3-12. 
261 Webb, E. K. (1969). “The temperature structure of the lower atmosphere.” Proc. Conf. 262 Refraction Effects in Geodesy and Conf. Electronic Distance Measurement, University of 263 New South Wales, Sydney, 1-9. 


\section{Figure captions}

265 Fig. 1. Refraction coefficient variation along the day: values computed from reciprocal

266 simultaneous observations Eq. (11) (solid line) versus values obtained by model Eq. (23)

267 (curved dotted line). The standard value $k=0.13$ is also represented (horizontal dotted line). 


\section{Tables}

269 Table 1. Refraction coefficients $k$ and refraction angles $\delta$ for different zenith angles at station 270 A, chord lengths and reference refraction values $(k=-0.15$ and $k=0.40)$ using: (1) Tsoulis et 271 al (2008) iterative method, (2) compact Eq. (11), and (3) the commonly used approximate 272 expression in Eq. (12).

273

\begin{tabular}{|c|c|c|c|c|c|c|c|c|c|c|c|c|c|c|c|c|c|c|c|c|}
\hline $\mathrm{Z}$ & deg) & $\begin{array}{l}\text { Length } \\
\text { (m) }\end{array}$ & 1) & 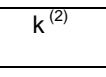 & 3) & $\begin{array}{l}\delta^{(1)} \\
\text { (cc) }\end{array}$ & $\begin{array}{l}\delta^{(2)} \\
\text { (cc) }\end{array}$ & $\begin{array}{l}\delta^{(3)} \\
\text { (cc) }\end{array}$ & $\begin{array}{c}\delta^{(1)} \\
(\text { (') }\end{array}$ & $\begin{array}{c}\delta^{(2)} \\
(")\end{array}$ & $\begin{array}{c}\delta^{(3)} \\
(')\end{array}$ & $\mathrm{k}^{(1)}$ & n & 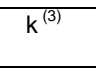 & $\begin{array}{l}\delta^{(1)} \\
(\mathrm{cc})\end{array}$ & $\begin{array}{l}\delta^{(2)} \\
(\mathrm{cc})\end{array}$ & $\begin{array}{l}\delta^{(3)} \\
(\mathrm{cc})\end{array}$ & $\begin{array}{l}\delta^{(1)} \\
\left({ }^{\prime \prime}\right)\end{array}$ & $\begin{array}{c}\delta^{(2)} \\
(")\end{array}$ & $\begin{array}{l}0 \\
\text { (') }\end{array}$ \\
\hline 55 & & & & & & 0 & & 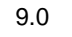 & & 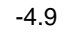 & 2.9 & & & & & & & .0 & 13.0 & \\
\hline 55 & 49.5 & 4000 & -0.1500 & 3 & 9 & 30.0 & 0.0 & 3.0 & -9.7 & -9.7 & 5.8 & 4000 & 2 & 0 & 0 & 0 & 9 & 9 & 9 & 41.4 \\
\hline 55 & 49.5 & 000 & 0 & 3 & ( & 45.0 & 4.9 & 7.0 & 14.6 & 4.5 & .8 & & & & .9 & .0 & & .9 & 8.9 & 62.2 \\
\hline 5 & & 8000 & & & & 50.0 & 8 & .1 & -19 & .4 & .7 & & & & & & & 8 & 1.8 & 2.9 \\
\hline 70 & 63 & 2000 & 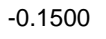 & . & & -15.0 & 5.0 & -4.1 & -4.9 & 10 & -1.3 & & & & 40.0 & 40.0 & 50.9 & 0 & .0 & 16.5 \\
\hline 70 & 63 & 80 & 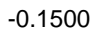 & . & 7 & -30.0 & 30.0 & 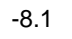 & -9.7 & . & -2.6 & & & & 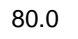 & . & & 25.9 & 5.9 & . \\
\hline 70 & 63 & 000 & & 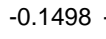 & & -45.0 & 9 & & -14.6 & 4.6 & -3.9 & & & & & & & 9 & 8.9 & 19 \\
\hline 10 & 0 & (100 & & & & -60.0 & 9.9 & -16.2 & -19.4 & 19.4 & -5.2 & & & & 9 & 60.0 & 6 & 8 & 1.8 & 56.0 \\
\hline 8 & (U) & 2000 & 0 & 0 & 23 & -15.0 & 15.0 & -12.2 & 4.9 & T. & 1.0 & & & & 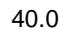 & 40.0 & & 0 & .0 & 13.8 \\
\hline 8 & 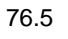 & 00 & & 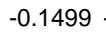 & 2 & -30.0 & 30.0 & -24 & 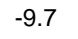 & 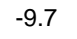 & -7.9 & & & & 80.0 & 8 & & 9 & 9 & 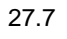 \\
\hline 8 & & 000 & & . & & -45.0 & 14.9 & -36.6 & -14.6 & 14.6 & -11.9 & & & & 9 & .0 & & 38.9 & 8.9 & 41.6 \\
\hline 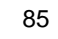 & & 8000 & & 9 & & -60.0 & 59.9 & -48.8 & 19.4 & 9.4 & -15 & & & & .9 & 0 & & 8 & 8 & 55.4 \\
\hline - & 90 & - & 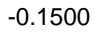 & . & 0 & - & 5.0 & - & -4.9 & . & . & & & & 40.0 & 40.0 & & 13.0 & 13.0 & 13.0 \\
\hline 100 & & 00 & & 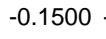 & & -30.0 & 30.0 & -30.0 & -9.7 & -9.7 & -9. & & & & 0 & & & $y$ & 9 & 25.9 \\
\hline 100 & 90 & 00 & & . & & -45.0 & 45.0 & -45.0 & -14.6 & 14.6 & -14 & & & & 9 & & & 9 & .9 & 8.9 \\
\hline 年 & & 8000 & & . & - & -60.0 & 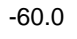 & - & - & 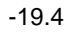 & $I_{1}$ & & & & 159.9 & 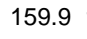 & & 51.8 & 51.8 & U. \\
\hline & & 000 & & . & 24 & -15.0 & 15.0 & -12.2 & -49 & -4 & -4.0 & & & & 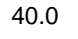 & & & 0 & 0 & 13 \\
\hline 11 & & 000 & & . & & -30.0 & -30.0 & -24.5 & -9.7 & -9.7 & -7.9 & & & & 80.0 & 19.9 & 85.5 & 25.9 & 5.9 & 27.7 \\
\hline & & קח & & 0.1501 & & -45.0 & -45.0 & -36.8 & 146 & 14.6 & -11.9 & & & & & & & & 38.8 & 41.0 \\
\hline$\perp$ & 1035 & בר & & . & 27 & - & 0 & -4 & 4 & 4 & -1 & & & & 9 & 8 & & 8 & 8 & 55.4 \\
\hline 130 & .7 & 2000 & & 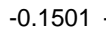 & & -15.0 & -15.0 & -4.1 & -4.9 & -4.9 & -1.3 & & & & 40.0 & 4 & & 0 & .0 & 16. \\
\hline 130 & $1+\pi$ & 7000 & & & & -30.0 & -30.0 & -8.3 & 6.1 & 0.1 & -2. & & & & & & & & 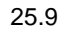 & 00 \\
\hline 130 & 21 & 6000 & & 2 & -0 & 450 & 5.0 & - & -14.6 & -14.6 & -4.0 & & & & 9 & & & 9 & 8 & 70.4 \\
\hline 130 & 117 & 0 & & 3 & -0 & -60.0 & 0.1 & -16.6 & -19.4 & -19.5 & -5.4 & & & & 159.9 & 15 & 3 & 51.8 & .8 & 65.9 \\
\hline & 0.5 & 000 & & & & -15.0 & -15.0 & & 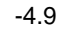 & & & & & & & & & & & $\angle 0$ \\
\hline 145 & 130.5 & 4000 & -0.1500 & 2 & 0 & -30.0 & 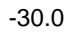 & 17.8 & . & -9.1 & 0.0 & & & & 80.0 & 79.9 & 8 & 9 & 9 & 41.4 \\
\hline 14 & 5 & 0 & -0.1500 & 2 & 0 & 0 & 5.0 & 7 & -14.6 & -14.6 & 8.7 & & & & 9 & 119.9 & & 9 & 8 & 62.1 \\
\hline+5 & 30.5 & 00 & 00 & 03 & 0889 & 60.0 & 60.1 & 35.5 & -19.4 & -19.5 & 11.5 & 00 & 97 & 91 & 159.9 & 159.8 & 255.5 & 51.8 & 51.8 & 82.8 \\
\hline
\end{tabular}

274 Note: $1 \mathrm{cc}=0.0001$ gon 


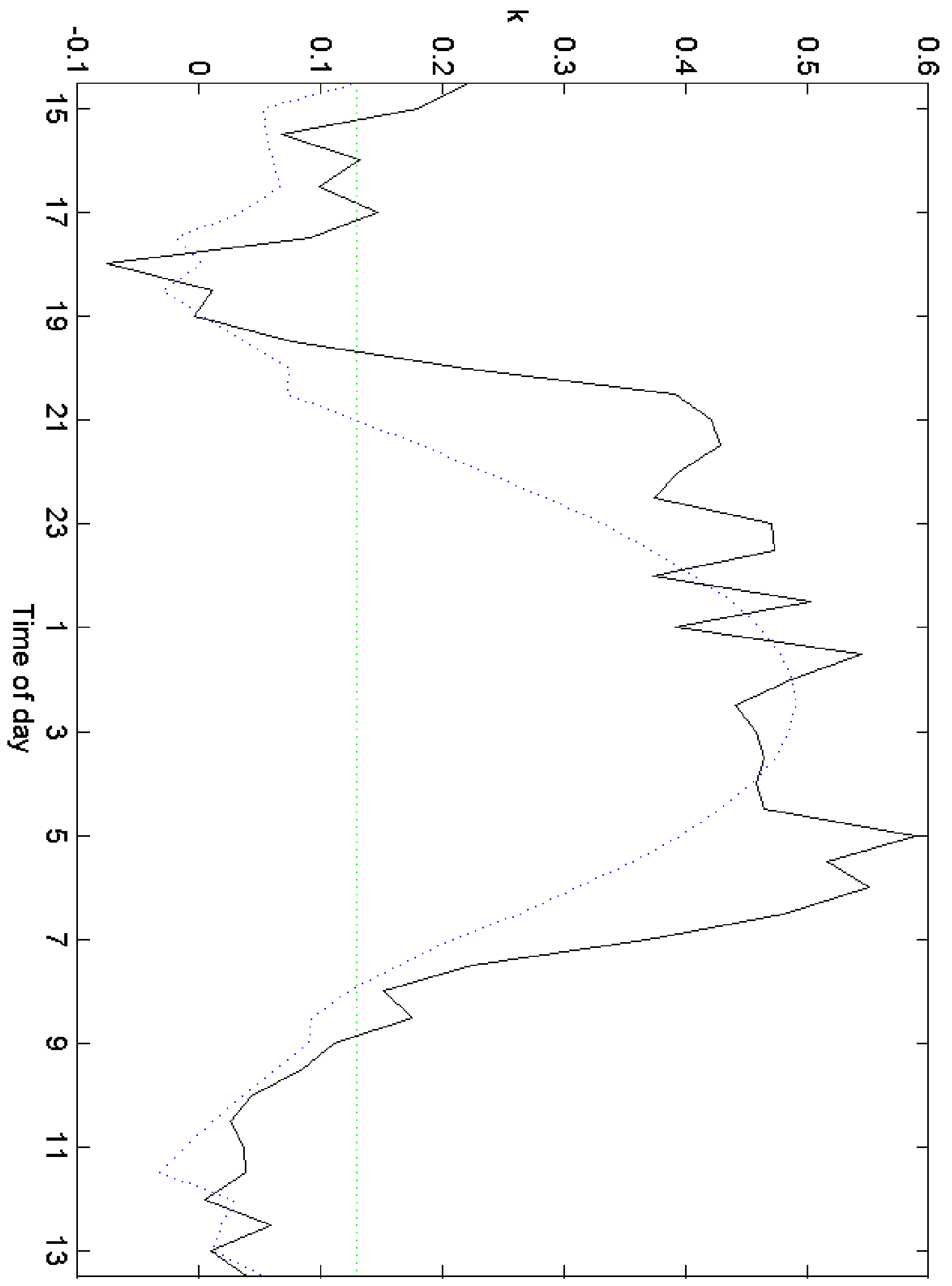

